ISSN: 0212-5374

DOI: http://dx.doi.org/10.14201/et20143215978

\title{
INCIDENCIA DE LAS CARPETAS DE APRENDIZAJE EN EL RENDIMIENTO ACADÉMICO DE LOS ESTUDIANTES UNIVERSITARIOS
}

\author{
Incidence of portfolio in the academic performance \\ of college students
}

Consequences de la méthodologie du dossier d'apprentissage sur le rendement académique des étudiants universitaires

\author{
Magda CAYÓn COSTA* y Marta Fuentes Agustí** \\ * Departamento de Economía de la Empresa de la Universidad Autónoma de \\ Barcelona.Correo-e: magda.cayon@uab.ca \\ ** Departamento de Psicología Básica, Evolutiva y de la Educación. Universidad \\ Autónoma de Barcelona.Correo-e: marta.fuentes@uab.cat
}

Recibido: 30-04-2013; Aceptado: 22-10-2013; Publicado: 30-03-2014

BIBLID [0212-5374 (2014) 32, 1; 59-78]

Ref. Bibl. MAGDA CAYÓN COSTA y MARTA FUENTES AGUSTÍ. Incidencia de las carpetas de aprendizaje en el rendimiento académico de los estudiantes universitarios. Enseñanza \& Teaching, 32, 1-2014, 59-78.

RESUMEN: Este trabajo pretende dar respuesta a las inquietudes detectadas en el debate sobre si los resultados académicos de los estudiantes mejoran con la introducción de nuevas metodologías docentes, cuestión que se plantea en diferentes foros sobre innovación docente universitaria. En concreto, se analiza la evolución de las notas obtenidas por los estudiantes de cinco asignaturas diferentes en diversas facultades en las que se ha introducido la carpeta del estudiante o portfolio como nueva 
MAGDA CAYÓN COSTA Y MARTA FUENTES AGUSTÍ

INCIDENCIA DE LAS CARPETAS DE APRENDIZAJE EN EL RENDIMIENTO ACADÉMICO DE LOS ESTUDIANTES UNIVERSITARIOS

metodología de enseñanza-aprendizaje. Se han comparado los resultados obtenidos antes y después de la implementación de la nueva metodología y se ha analizado si existe robustez estadística en la diferencia de dichos resultados. Asimismo, se ha comparado la evolución de la carpeta del estudiante, una vez ha sido implantada, en cuanto a los resultados obtenidos para establecer la repercusión de los cambios introducidos paulatinamente respecto a la carpeta propuesta inicialmente. Los datos obtenidos confirman que, en los casos estudiados, los resultados académicos de los estudiantes mejoran claramente. Se reducen drásticamente los suspendidos, y el nivel mayoritario se sitúa en el notable.

Palabras clave: aprendizaje significativo, innovación didáctica, carpeta del estudiante, universidad, enseñanza reflexiva.

SUMMARY: This paper aims to address the concerns about whether students' academic results improve with the introduction of new teaching methodologies. This is a recurrent issue in the specialised forums devoted to teaching innovation at college level. In particular, we analyze the evolution of the marks obtained by the students of five different subjects related to diverse degrees, where the student's portfolio have been introduced as a new methodology and following common guidelines. It has been compared the marks before and after the introduction of the new methodology and they have been analyzed to establish if the difference in these results presented statistical robustness. We also compared the progression in the design of the student's portfolio proposed by the professors during its implementation. The results obtained confirm that the academic performance of students clearly improves in the cases analysed, dramatically reducing the fails, and improving remarkably the proportion of merit marks.

Key words: meaningful learning, educational innovation, student, University, student's portfolio.

RÉSUMÉ: Ce travail vise à répondre aux préoccupations soulevées dans le débat quant à savoir si les résultats académiques des élèves s'amélioré avec l'introduction des nouvelles méthodologies d'enseignement proposées dans divers forums sur l'innovation de l'enseignement universitaire. Plus précisément, nous analysons l'évolution des scores obtenus par les élèves par rapport à cinq sujets différents et provenant de différentes facultés où sont inscrits, en prenant en considération l'introduction du dossier ou portfolio de l'élève en tant que nouvelle méthode d'enseignement-apprentissage. Nous avons comparé les résultats obtenus avant et après la mise en ouvre de la nouvelle méthodologie et analysé s'il y a une différence et une robustesse statistique de ces résultats. En outre, nous avons comparé l'évolution du dossier de l'étudiant, une fois qu'il a été mis en place, comme les résultats d'établir l'impact des changements introduits progressivement sur le dossier initialement proposé. Les données obtenues confirment que, dans les cas étudiés, le rendement scolaire des élèves se sont nettement améliorées. Réduisant 
MAGDA CAYÓN COSTA Y MARTA FUENTES AGUSTÍ

INCIDENCIA DE LAS CARPETAS DE APRENDIZAJE EN EL RENDIMIENTO ACADÉMICO DE LOS ESTUDIANTES UNIVERSITARIOS

considérablement les échecs, et le niveau de la majorité dépasse les niveaux intermédiaires par rapport aux résultats scolaires.

Mots clés: apprentissage significatif, l'innovation pédagogique, portfolio de l'élève, le collège, l'enseignement réflexif.

\section{INTRODUCCIÓN Y ESTADO DE LA CUESTIÓN}

La carpeta de aprendizaje es uno de los métodos que se puede utilizar en educación superior para evaluar los aprendizajes de los estudiantes, ya que permite una gran adaptabilidad a las necesidades de los contextos y situaciones existentes. Es un método centrado en los aprendizajes del alumnado. Es una herramienta útil que permite recopilar múltiples evidencias que ilustran los esfuerzos y progresos del estudiante en su proceso de formación (GI-CAES, 2008).

\subsection{Metodologías de enseñanza-aprendizaje}

La tarea docente en general y la universitaria en particular está experimentando un cambio de paradigma. Los estudiantes están conviviendo en una sociedad muy distinta, en todos los sentidos, a la de hace unos años. Entre otras cosas, la facilidad de acceder a la información que actualmente existe les permite disponer de todos los contenidos inimaginables al momento, y esto hace que el rol del docente deba cambiar radicalmente respecto al profesor universitario clásico basado en clases magistrales. En relación a este cambio del docente existen diferentes investigaciones, como la de Villar y Alegre (2005), que afirman que "cuando el profesorado percibe que la docencia, la organización material y el contenido, las técnicas docentes innovadoras, o la interacción estudiante profesor se asocian con los resultados obtenidos en los estudiantes, entonces reconoce la conveniencia de promover mecanismos de evaluación y mejora de su docencia práctica...".

El problema de investigación que motiva este trabajo es la inquietud que presenta el profesorado sobre cómo asegurar que el aprendizaje de los estudiantes universitarios sea real y duradero; y para ello se plantea si el uso de determinadas metodologías de enseñanza-aprendizaje-evaluación mejora el rendimiento académico. En concreto, se analiza la incidencia de la Carpeta de Aprendizaje o Carpeta del Estudiante.

El estudiante debe aprender conceptos, procesos y actitudes, desarrollar habilidades, y sobre todo responsabilizarse de su propio aprendizaje, de su propio progreso y tomar consciencia del proceso de aprendizaje que está experimentando. En definitiva, en la Universidad se deben formar personas en un amplio espectro y construir las asignaturas en aras de conseguirlo, tanto desde el punto de vista de contenidos como de actividades propuestas encaradas a poder asegurar dicho aprendizaje. Y lo más importante será elegir la metodología (Pozo y Monereo, 1999; Villar, 2004; Villar y Alegre, 2008) más adecuada a cada materia y contexto, 
MAGDA CAYÓN COSTA Y MARTA FUENTES AGUSTÍ

INCIDENCIA DE LAS CARPETAS DE APRENDIZAJE EN EL RENDIMIENTO ACADÉMICO

DE LOS ESTUDIANTES UNIVERSITARIOS

así como establecer un vínculo de comunicación profesor-estudiante para poder periódicamente valorar el estado en el que se encuentra el aprendizaje propuesto.

Una de las metodologías (Barbera, 2005; Bia, 2005; Blanch y otros, 2008) ampliamente aceptada para el desarrollo de este tipo de docencia es la Carpeta de Aprendizaje del Estudiante (CAEs) o portfolio del estudiante ${ }^{1}$, con la que el estudiante se sitúa en el centro del proceso formativo. La carpeta de aprendizaje es un sistema de formación muy versátil pero con unos criterios de desarrollo y evaluación muy claros y fijados al principio del proceso, que atribuyen al estudiante universitario la responsabilidad de su propia evaluación y de su propio aprendizaje. El uso de la carpeta como eje vertebrador de la enseñanza, aprendizaje y evaluación del estudiante exige una interpretación constructiva de este aprendizaje y un abordaje docente basado en la necesidad de ofrecer a los estudiantes experiencias tan ricas y variadas como sea posible, además de una concepción de la evaluación como actividad para la mejora del aprendizaje y la comprobación de resultados (proceso y progreso).

La carpeta de aprendizaje permite evaluar conocimientos, estrategias cognitivas y disposiciones hacia el aprendizaje. Desde esta perspectiva, la evaluación durante el uso de carpetas se entiende de forma compleja, no lineal y multidimensional, y adquiere especial relevancia la evaluación de los procesos de reflexión, narración y reconstrucción conscientes de los aprendizajes.

Con esta metodología, la evaluación se integra en el proceso de enseñanzaaprendizaje. Por esta razón, docentes y estudiantes pasan a compartir diferentes momentos y formas de evaluación durante el desarrollo de la carpeta: evaluación inicial (diagnóstica), evaluación continuada (seguimiento por parte del profesor), coevaluación (evaluación entre estudiantes), autoevaluación (del propio estudiante) y evaluación final (compendio de todas ellas). Lo que diferencia la metodología CAES de cualquier otra es que compromete al estudiante a evidenciar la reflexión sobre su propio proceso y progreso en el aprendizaje en todas y cada una de las actividades que desarrolla. Al mismo tiempo, exige una retroalimentación por parte del docente, y en la mayoría de los casos, la de sus compañeros.

El portfolio del estudiante promueve el aprendizaje basado en problemas, el trabajo cooperativo y facilita la adquisición de competencias transversales especificadas en el nuevo contexto del EEES. Al aplicarlo, cada uno, tanto el profesor como el estudiante, hace una aportación personal. Al primero, le comporta una

1. Mantenemos el término inglés portfolio por el amplio consenso que presenta en las diferentes prácticas que aglutina y con el ánimo de evitar las distintas traducciones en los diferentes idiomas que en la mayor parte de casos son todavía más polisemias y menos exclusivas (carpetas, dosier de aprendizaje, por ejemplo). También se considera que éste es un término compartido en los campos tecnológico, empresarial y educativo. Se ha optado por la vertiente electrónica (e-portfolio) por su versatilidad, ubicuidad, interoperatividad, posibilidad de acceso múltiple y compartido. Por lo que nos referiremos indistintamente a portfolio y e-portfolio. 
serie de decisiones que marcan su futuro como profesional de la educación en la universidad y, al segundo, le aporta aprendizaje (a corto y largo plazo), evaluación (reflejada en el expediente académico) y un modo de aprender a aprender.

\subsection{Impacto del portfolio del estudiante}

En estos momentos de cambio en el entorno universitario europeo, se habla del sistema del portfolio del estudiante por doquier, como para conseguir la mejora de la calidad docente, Pero esta metodología no es nueva, ya en 1991 D. W. Johnson, R. T. Johnson y K. A. Smith comentan que los estudiantes se vuelven mucho más sofisticados y competentes cuando pueden organizar su trabajo en un portfolio que representa la calidad de su aprendizaje en un curso o un año de la universidad. No hay sustituto para que los estudiantes recopilen y organicen sus muestras de trabajo y puedan expresar una lógica conexión de dichas muestras en una visión completa y global de sus logros, su desarrollo y su crecimiento como futuros profesionales.

La literatura más especializada en las carpetas de aprendizaje, como método de aprendizaje y evaluación, plantea algunos objetivos para promover su implementación en el proceso de enseñanza-aprendizaje en la educación superior. Entre ellos cabe resaltar:

- Observar el progreso y el proceso seguido durante el aprendizaje, tanto por el profesor como para el estudiante (Farr y Tone, 1994; Chatel, 2001; Fuentes y Oliver, 2008).

- Promover el aprendizaje autónomo del estudiante (Martínez, Bordons, Guarro y Manuel, 2006) facilitándole aprender a aprender y a reflexionar sobre los propios errores.

- Individualizar el aprendizaje del estudiante (Barret, 2004; Hernández Fernández, 2005; Fuentes y Oliver, 2008).

- Comprometer al estudiante en su propia evaluación, ya que este conoce los objetivos y los criterios de evaluación y mantiene un diálogo constante con el profesor sobre su propio aprendizaje (Sunstein y Lovell, 2000; Alegret, Laguna, Vázquez y Sánchez, 2006).

- Demostrar el nivel de competencia y el grado de profundización sobre los aprendizajes y validar los métodos y las técnicas de enseñanza para facilitar y favorecerlos (Klenowski, 2004; Gimeno, González y Santiveri, 2008).

- Proporcionar al profesor información sobre el aprendizaje y la evaluación de manera más diversificada y desde diferentes perspectivas (Marby, 1999; Johnson, Mims-Cox y Doyle-Nichols, 2006).

- Fomentar la capacidad de reflexionar sobre el propio aprendizaje, la forma de comunicar sus logros y estrategias de compilación de la información (Ugalde y López, 1995; Colén, Giné y Imbernon, 2006; Fuentes, Oliver, Jimenez, Segura y González, 2009). 
- Desarrollar la reflexión (revisión crítica y práctica reflexiva de los procesos y prácticas de aprendizaje) (Brookfield, 1995; Castro Quitora, 2002).

- Evaluar mediante la retroalimentación (Colén, Giné y Imbernon, 2006).

- Introducir la autoevaluación y la metacognición en el proceso de aprendizaje (Hacker, 1998; Watkins, 2001; Klenowski, 2004; Zubizarreta, 2004) y evaluación (Cerioni, 1997; Blanch et al., 2009).

Fuentes y Oliver (2008, 2010), además de argumentar lo enunciado en párrafos anteriores, recogen qué significa para el profesorado la aplicación de la CAEs que de manera sintética podemos resumir en: una reformulación en profundidad de la metodología didáctica, un proceso de reflexión continuo sobre su práctica docente, una mayor gestión y organización de los elementos que acreditan el aprendizaje del estudiante, un saber compartir y hacer visible el proceso de enseñanza-aprendizaje y del sistema de evaluación, una formación continuada en recursos y estrategias didácticas para poder incorporar una mayor diversidad de soportes y de formatos en la presentación, la elaboración y la evaluación de la CAES, adoptar una evaluación continua siendo la retroalimentación cualitativa una constante, un replanteamiento global de las titulaciones, una mayor coordinación entre el profesorado y un mayor diálogo e intercambio con los estudiantes.

Así mismo resaltan lo que el uso del portfolio del estudiante ha significado para el propio estudiante: un aumento significativo de su participación en el aula, una mayor concienciación de su propio proceso de aprendizaje, un aumento del compromiso con el propio aprendizaje, un uso e incorporación de nuevos recursos didácticos y tecnológicos que favorecen y facilitan el desarrollo de competencias transversales y específicas de la asignatura, una construcción conjunta del conocimiento en la medida que permite compartir materiales, reflexionar y coordinarse con diversos grupos de estudiantes, una evaluación compartida y más justa incorporando la evaluación externa del profesor, la coevaluación entre compañeros y la autoevaluación.

\subsection{Objetivo}

El objetivo general de este trabajo es evidenciar empíricamente si los resultados en el aprendizaje universitario mejoran utilizando la metodología de la carpeta del estudiante.

A continuación se exponen los principales antecedentes que han motivado esta investigación.

En la Universidad Autónoma de Barcelona se fomentan los denominados Grupos de Interés (GI) en innovación docente en diferentes áreas. Concretamente el GI-CAES ${ }^{2}$ está formado por docentes de diferentes áreas y departamentos que de forma global o parcial están aplicando el portfolio del estudiante en sus asignaturas

2. http://www.caeslav.com. 
de grado siguiendo criterios comunes. Es un grupo dinámico que, en sus reuniones mensuales, pone en común experiencias, inquietudes y dificultades en la aplicación de la CAEs, ayudando a sus miembros a mejorar su práctica docente diaria y los cursos y talleres que imparten sobre la aplicación del portfolio del estudiante en el entorno universitario. En dichas formaciones/cursos suele darse la constante de que los docentes asistentes pregunten sobre evidencias de las mejorías observadas en los resultados académicos de los estudiantes. Cuestión que también se debate en la Red española e-portfolio ${ }^{3}$ y en el proyecto-convenio con la Universidad Nacional del Comahue (Argentina) que tiene el grupo ${ }^{4}$.

También cabe destacar que en los diferentes congresos sobre innovación docente en los que se ha participado, así como en la bibliografía revisada, se habla mucho de las diferentes metodologías docentes aplicadas, de las diferentes maneras de evaluar, etc., pero se detecta un vacío en cuanto a la presentación de resultados que avalen la puesta en marcha de estas innovaciones expuestas.

Por todo lo anterior, se ha planteado dar respuesta a estas inquietudes y hacer balance global de los resultados académicos en diferentes asignaturas de grado donde se ha aplicado la carpeta del estudiante.

Para ello se persiguen los siguientes objetivos específicos:

1. Analizar la incidencia de la implantación de la CAES en el rendimiento académico (pre-post CAEs).

2. Analizar la incidencia que las aportaciones de la experiencia docente y estudiantil en el uso de la CAEs han tenido en el rendimiento académico.

\section{Metodología}

Existe un estudio previo donde se recopilaron las opiniones y voces de los estudiantes y profesores de diversas titulaciones de la Facultad de Ciencias de la Educación de la UAB mediante un cuestionario y varias entrevistas y narrativas digitales sobre el uso del portfolio del estudiante (Blanch et al., 2010). Partiendo de este antecedente, en el presente estudio se pretende analizar la incidencia de la CAEs en el rendimiento académico de los estudiantes.

Para medir el éxito en el aprendizaje, la variable más objetiva de la que se dispone en educación son las calificaciones obtenidas por los estudiantes. Por lo que para conseguir el objetivo propuesto se plantea un análisis en dos direcciones:

3. Red nacional que aglutina más de dieciséis equipos de investigación de diferentes universidades nacionales y siete empresas y se encarga, entre otras cosas, de impulsar un observatorio permanente sobre el nivel de presencia y actividad de iniciativas desarrolladas en las universidades con la herramienta del portfolio del estudiante: http://www.redportfolio.org/.

4. El grupo GI-CAES asesora a la Universidad Nacional del Comahue en la aplicación de la CAES en su docencia. 
a. Por un lado, para determinar cómo ha mejorado el aprendizaje con la implantación de la carpeta se han comparado los resultados obtenidos por los alumnos en el curso académico en que se ha introducido la nueva metodología con el curso anterior en que se utilizaba otra metodología. Posteriormente se comprueba la robustez de los resultados a través de la prueba t para la diferencia de medias de muestras no emparejadas para determinar si existen diferencias estadísticamente significativas.

b. Y, por otro lado, para analizar el éxito en el aprendizaje del estudiante en el uso tipo de metodología docente, se han comparado los resultados obtenidos en los sucesivos cursos de aplicación de la carpeta del estudiante y se ha entrevistado a los profesores implicados para la correcta interpretación de las posibles variaciones.

TABLA 1

Asignaturas que han colaborado en el presente estudio

\begin{tabular}{|c|c|c|c|c|}
\hline TitulaCióN & ASIGNATURA & CRÉDITOS & $\begin{array}{c}\mathrm{N} . \stackrel{\circ}{ } \\
\text { ESTUDIANTES }\end{array}$ & \begin{tabular}{|c|} 
CURSO \\
ACADÉMICO \\
\end{tabular} \\
\hline $\begin{array}{l}\text { Ingeniero técnico industrial, } \\
\text { especialidad en química } \\
\text { industrial }\end{array}$ & $\begin{array}{l}\text { Evaluación del } \\
\text { impacto ambiental } \\
\text { y auditorías }\end{array}$ & 4,5 & $\begin{array}{l}17 \\
20 \\
16 \\
31 \\
25\end{array}$ & \begin{tabular}{|l|}
$2006-2007$ \\
$2007-2008^{*}$ \\
$2008-2009 *$ \\
$2009-2010^{*}$ \\
$2010-2011^{*}$
\end{tabular} \\
\hline $\begin{array}{l}\text { Graduado en Dirección } \\
\text { Hotelera }\end{array}$ & $\begin{array}{l}\text { Dirección estratégica } \\
\text { y financiera de la } \\
\text { empresa }\end{array}$ & 10,5 & $\begin{array}{l}68 \\
64 \\
70 \\
85 \\
83\end{array}$ & $\begin{array}{l}2006-2007 \\
2007-2008 \\
2008-2009^{*} \\
2009-2010^{*} \\
2010-2011^{*}\end{array}$ \\
\hline $\begin{array}{l}\text { Magisterio, Especialidad en } \\
\text { Educación Primaria }\end{array}$ & $\begin{array}{l}\text { Ciencias Sociales } \\
\text { y su didáctica I }\end{array}$ & 4 & $\begin{array}{l}47 \\
45 \\
30 \\
27 \\
25 \\
38\end{array}$ & $\begin{array}{l}2004-2005 \\
2005-2006^{*} \\
2006-2007^{*} \\
2007-2008^{*} \\
2008-2009^{*} \\
2009-2010^{*}\end{array}$ \\
\hline $\begin{array}{l}\text { Magisterio, Especialidad en } \\
\text { Educación Primaria }\end{array}$ & $\begin{array}{l}\text { Psicología Evolutiva } \\
\text { y de la Educación }\end{array}$ & 8 & $\begin{array}{l}73 \\
85 \\
34 \\
79 \\
71 \\
\end{array}$ & \begin{tabular}{|l|}
$2006-2007$ \\
$2007-2008$ \\
$2008-2009 *$ \\
$2009-2010^{*}$ \\
$2010-2011^{*}$
\end{tabular} \\
\hline $\begin{array}{l}\text { Magisterio, Especialidad en } \\
\text { Educación Infantil }\end{array}$ & $\begin{array}{l}\text { Atención a la primera } \\
\text { infancia }\end{array}$ & 6 & $\begin{array}{l}46 \\
50 \\
59 \\
56 \\
58\end{array}$ & $\begin{array}{l}2005-2006^{*} \\
2006-2007^{*} \\
2007-2008^{*} \\
2008-200{ }^{*} \\
2009-2010^{*}\end{array}$ \\
\hline
\end{tabular}

* Los cursos marcados son los que han aplicado la carpeta de aprendizaje. 
Los datos se han obtenido a partir de fuentes primarias. Para obtener los datos cuantitativos se han utilizado las notas finales publicadas en las actas oficiales en 5 asignaturas diferentes y de titulaciones distintas. Paralelamente se han llevado a cabo entrevistas en profundidad al profesorado de dichas asignaturas con el objetivo de obtener de primera mano las reflexiones de las experiencias de los docentes para el análisis exhaustivo de los datos a lo largo de la aplicación de la CAES en sus asignaturas.

Todas las asignaturas de la muestra pertenecen a licenciatura o grado acorde con el mapa de la Red e-portfolio, que muestra que el 84,4\% de aplicaciones del portfolio en la universidad se da en el nivel de grado, un 9,4\% en el doctorado y un $6,2 \%$ en postgrado.

En todas ellas la carpeta empieza al inicio de la asignatura. En ese momento se explicita a los estudiantes el plan de trabajo. El profesorado les propone trabajar mediante carpetas, herramienta que servirá para el aprendizaje del estudiante y el seguimiento por parte del profesor del progreso y proceso de sus discentes. Por consiguiente, se pacta que la carpeta se convierta en el principal elemento de construcción conjunta del conocimiento y de evaluación formativa y continuada del estudiante. Así como el producto de la evaluación sumativa.

De los 5 casos analizados en 4 la carpeta supone el 100\% de la evaluación final. Estas incluyen diferentes tipos de evidencias, desde las que son fruto de las actividades de enseñanza-aprendizaje propuestas por el profesorado hasta las resultantes de la iniciativa de un estudiante o grupo de estudiantes. Desde las que responden a un aprendizaje dirigido a las actividades autónomas, a las que parten de actividades realizadas en gran grupo (exposición en el aula, debates, etc.), en grupos reducidos (trabajo cooperativo, análisis de casos, etc.) o individuales, o desde las iniciadas a las realizadas totalmente o en parte en el aula presencial y/o en el entorno virtual, etc.

El profesorado marca y pauta la construcción de la carpeta al mismo tiempo que cada estudiante define el diseño, estructura, etc., de su carpeta de aprendizaje, por lo que en este proceso las idas y venidas de lo aprendido son fundamentales para lograr un progreso en la línea de mejora continua. Llegando a elaborar una gran diversidad de carpetas de aprendizaje ya que cada una de ellas es individual y única porque cada estudiante es distinto y único, y aprende cosas distintas y de diferente forma.

En el recorrido formativo la esencia de la carpeta se ha mantenido pero el índice, el formato, la presentación, los documentos de soporte, el material de guía, etc., han ido variando. Entre estos cambios resaltamos el uso de un entorno virtual como apoyo a las clases presenciales. En concreto, en las experiencias analizadas se ha introducido el uso del Campus Virtual propio de la UAB. A pesar de sus limitaciones, sobre las que se va avanzando gracias a la comunicación establecida entre el profesorado y la Oficina de la Autónoma Interactiva Docente (OAID), el entorno virtual facilita: compartir archivos; iniciar foros y debates; compartir a modo de ejemplo evidencias de aprendizaje con los compañeros; realizar 
coevaluaciones formativas que ayudan al aprendizaje y mejoran el producto final; el seguimiento y revisión de las carpetas; la organización de la información y su gestión; actúa de repositorio; guía la construcción de la carpeta y ayuda a tener una carpeta actualizada (al día); proporciona hábitos de revisión y autorregulación del propio trabajo y de supervisión interna y externa; y también facilita la comunicación entre los estudiantes y entre profesor y estudiante, agiliza la gestión del tiempo y posibilita dar respuestas inmediatas a dudas e inquietudes.

Los recursos del entorno y, sobre todo, el uso que propone el profesorado son la clave para un desarrollo adecuado de la innovación docente presentada. En nuestro caso, el diseño y la estructura base del entorno se restringe al campus virtual genérico de la UAB donde el grado de libertad es limitado. Al igual que en el aula presencial, es el profesorado quien a partir de una infraestructura y mobiliario concreto debe hacer propuestas didácticas que se puedan llevar a la práctica.

La carpeta final es presentada individualmente o en grupo, en formato papel o electrónico con el conjunto de evidencias obligatorias (comunes en todas las carpetas y seleccionadas algunas por iniciativa del profesorado y otros del grupo clase), optativas (elegidas por cada uno de los estudiantes entre las propuestas que han ido surgiendo en el desarrollo de la asignatura) y voluntarias (surgidas por iniciativa individual y que generalmente dan respuesta a perfiles concretos e intereses muy particulares del estudiante).

\section{ANÁlisis de los Resultados}

Conforme lo comentado en la parte de metodología, se exponen los resultados en función de dicho planteamiento:

a. Comparación de las notas obtenidas por los alumnos en el curso de la introducción de la carpeta de aprendizaje con las notas del curso anterior. Estas notas presentan el formato español habitual de 0 a 10 puntos (los no presentados se han valorado como 0).

b. Comparación de las notas obtenidas por los alumnos en los cursos de aplicación de la CAES reflejando la posible incidencia de los cambios introducidos en su implementación (proceso de evaluación, socialización, experiencia acumulativa de uso, etc.).

\subsection{Comparativa de los resultados pre/post-CAES}

Empezando por el primer análisis comparativo, se presenta el siguiente cuadro de datos: la asignatura en cuestión, las notas promedio obtenidas por los estudiantes antes de la aplicación de la CAES y el curso posterior donde ya se aplicaba la CAEs; los resultados de la prueba de robustez, donde primero figura el valor del estadístico y entre paréntesis el nivel de significación; y finalmente el sistema de evaluación antes y después de la implantación de la carpeta. 
MAGDA CAYÓN COSTA Y MARTA FUENTES AGUSTÍ

INCIDENCIA DE LAS CARPETAS DE APRENDIZAJE EN EL RENDIMIENTO ACADÉMICO

DE LOS ESTUDIANTES UNIVERSITARIOS

TABLA 2

Comparación pre/post-CAES

1. EVALUACIÓN DEL IMPACTO AMBIENTAL Y AUDITORÍAS (FEBRERO-JUNIO)

\begin{tabular}{|l|l|}
\hline Nota promedio pre-CAES & \multicolumn{1}{c|}{3,37} \\
\hline Nota promedio post-CAES & \multicolumn{1}{c|}{-3,3 987 (sig. > 99\%) } \\
\hline Estadístico «t" & \multicolumn{1}{|c|}{ un trabajo (30\%) y un examen final (70\%) } \\
\hline Evaluación pre-CAES & $\begin{array}{l}5 \% \text { Carta de Presentación (Individual), 15\% Simulación de } \\
\text { Auditoría Ambiental: Caso práctico (Grupos aleatorios), 10\% } \\
\text { Presentación y redacción del informe parcial de auditoría } \\
\text { ambiental. (El mismo grupo que por la actividad anterior), 15\% } \\
\text { Redacción de un informe de auditoría global (Grupo definido } \\
\text { por el profesor), 10\% Ejercicios diversos, 25\% Presentación del } \\
\text { Estudio de Impacto Ambiental de una actividad elegida por los } \\
\text { alumnos (Grupos definidos por los mismos alumnos al inicio } \\
\text { de la asignatura), 10\% Cajón de-Sastre: cualquier otra evidencia } \\
\text { de aprendizaje que el alumno quiera incluir, 10\% Elaboración } \\
\text { Final de la carpeta de aprendizaje }\end{array}$ \\
\hline
\end{tabular}

Comentario: clara disminución de los NP, pasan del 46\% al 5\%. Disminuye el número de aprobados para situarse en el notable básicamente y algunos en el sobresaliente.

\begin{tabular}{l}
\hline \multicolumn{2}{|c|}{ 2. DIRECCIÓN ESTRATÉGICA Y FINANCIERA DE LA EMPRESA (SEPTIEMBRE-ENERO) } \\
\hline Nota promedio pre-CAES
\end{tabular}


MAGDA CAYÓN COSTA Y MARTA FUENTES AGUSTÍ INCIDENCIA DE LAS CARPETAS DE APRENDIZAJE EN EL RENDIMIENTO ACADÉMICO DE LOS ESTUDIANTES UNIVERSITARIOS

Lecturas obligatorias (15\%), actividades obligatorias (30\%), visita a La Garrotxa y su preparación en grupo (15\%), reflexiones individuales sobre las ciencias sociales y su didáctica (25\%),

Evaluación post-CAES reflexiones individuales sobre el trabajo cooperativo, la autoevaluación y el papel del docente (15\%), actividades opcionales individuales (10\%) de lecturas recomendadas y noticias de actualidad.

Comentario: disminución de un 10\% de los no presentados y de un $4 \%$ los suspendidos, situándose el $85 \%$ de los estudiantes en el aprobado. Desaparecen los notables y los sobresalientes.

\begin{tabular}{|l|l|}
\hline \multicolumn{2}{|c|}{ 4. PSICOLOGÍA EVOLUTIVA Y DE LA EDUCACIÓN (SEPTIEMBRE-JUNIO) } \\
\hline Nota promedio pre-CAES & \multicolumn{1}{|c|}{4,39} \\
\hline Nota promedio post-CAES & \multicolumn{1}{|c|}{6,07} \\
\hline Estadístico "t” & \multicolumn{1}{|c|}{$-2,434$ (sig. > 90\%) } \\
\hline Evaluación pre-CAES & $\begin{array}{l}60 \% \text { actividades individuales: estudios de caso, debates y } \\
\text { proyectos } \\
40 \% \text { actividades grupales: análisis de casos, debates y } \\
\text { proyectos }\end{array}$ \\
\hline Evaluación post-CAES & $\begin{array}{l}60 \% \text { evidencias individuales obligatorias y voluntarias } \\
40 \% \text { evidencias en grupo obligatorias y voluntarias }\end{array}$ \\
\hline $\begin{array}{l}\text { Comentario: los no presentados incrementan un 5\% y los notables un 7\%, en cambio, los } \\
\text { sobresalientes disminuyen un 4\%, y los aprobados un 11\%. }\end{array}$ \\
\hline
\end{tabular}

La quinta asignatura de la muestra no la incluimos en este primer análisis porque desde un principio se ha impartido utilizando carpetas de aprendizaje, con lo cual no tenemos años pre-CAES.

Resumen de los principales resultados anteriores:

1. En tres de los cuatro casos analizados el estadístico presenta una significatividad superior al 90\%, lo que viene a decir que los resultados académicos obtenidos por estudiantes antes de introducir la CAES y los obtenidos después de implantar CAES son significativamente distintos, y en este caso mejores (como se resalta en los comentarios). En el caso (segunda asignatura) que el estadístico no presenta significatividad, y por tanto que aparentemente no mejoran los resultados académicos, queda bastante claro que la metodología previa utilizada era muy próxima a lo que entendemos por CAEs, lo que provoca que el impacto sobre los resultados sea mucho menor o inapreciable.

2. Los sobresalientes disminuyen para aumentar el número de notables y aprobados. En todos los casos los suspendidos disminuyen drásticamente hasta desaparecer.

3. El profesorado comenta que en un primer momento (coincidiendo con la presentación de la asignatura), a los estudiantes les cuesta comprender 
MAGDA CAYÓN COSTA Y MARTA FUENTES AGUSTÍ DE LOS ESTUDIANTES UNIVERSITARIOS

la esencia de la CAES, se muestran sorprendidos por la enseñanza diversificada que supone, y al mismo tiempo con curiosidad por la manera en que deberán enfocar su estudio y en que serán evaluados y contentos de dejar atrás la palabra "examen".

\subsection{Comparativa de los resultados durante la evaluación de la CAES}

A continuación se presentan los resultados del segundo análisis hecho, que consiste en estudiar la evolución de los resultados durante los cursos en que se han aplicado carpetas. En la Tabla 3 se indica la asignatura, análisis del rendimiento académico de los estudiantes en general, y en el comentario del profesor, se explicitan los cambios introducidos en la aplicación de la CAEs.

TABLA 3

Comparación de los años de implantación de la CAES

\begin{tabular}{|c|c|}
\hline $\begin{array}{l}\text { 1. EVALUACIÓN } \\
\text { DEL IMPACTO } \\
\text { AMBIENTAL Y } \\
\text { AUDITORÍAS }\end{array}$ & $\begin{array}{l}\text { El segundo año de CAES no hay diferencia significativa en los } \\
\text { resultados académicos. } \\
\text { En el tercer y cuarto año de CAEs las diferencias son significativas, } \\
\text { por encima del } 95 \% \text {, pero de forma no homogénea. El tercer año } \\
\text { los alumnos se sitúan entre el notable y el excelente y el cuarto año } \\
\text { se sitúan entre el aprobado (mayoría) y el notable, incrementando } \\
\text { los NP. } \\
\text { Como evolución global se destaca la disminución muy importante } \\
\text { de los NP (pasando del } 46 \% \text { al } 10 \% \text { ) y la eliminación total de los } \\
\text { sobresalientes. }\end{array}$ \\
\hline
\end{tabular}

Comentario del profesor. incorporación del role playing, involucrar a otros actores, además del alumno y profesor, en las actividades, incorporación de la evaluación en la responsabilidad del alumno (co- y autoevaluación). Casos prácticos reales.

Las opiniones y percepciones de los alumnos se han recopilado a través de las respuestas a la siguiente pregunta: ¿Cuáles son las características de este profesor/curso que te han ayudado en el proceso de aprendizaje de este curso?:

- Su implicación y preocupación por el trabajo que íbamos haciendo ha sido fundamental.

- Me ha ayudado a ser constante y organizado.

- Me han gustado mucho las actividades de clase y el material de apoyo.

- Para el trabajo que supone, la asignatura debería tener más créditos.

- Me ha gustado la dinámica de la clase, discutir ideas y que se me tome en cuenta. 


\section{DIRECCIÓN} ESTRATÉGICA Y FINANCIERA DE LA EMPRESA
A pesar de que los años posteriores a la aplicación tampoco la diferencia de los resultados sea significativa, se observa mucha más homogeneidad que la diferencia entre el curso pre- y post-CAES. El nivel de suspendidos se mantiene y se observa migración de los aprobados hacia notables y no presentados, habiendo un número residual en la excelencia.

Comentario del profesor. La ausencia de significatividad estadística es básicamente debida a que la metodología utilizada previamente tenía mucha similitud al espíritu de la carpeta. Contando con diferentes tipos de actividades a desarrollar y utilización de distintos métodos de evaluación. Pero no era propiamente una carpeta ya que faltaba una parte esencial que era la reflexión continua del alumno durante todo el proceso y en todo lo que aprende, y la oportunidad de replantearse el trabajo hecho. Todo y con eso, entiendo que en este tipo de metodología es muy complicado llegar a la excelencia, ya que es muy difícil que el estudiante sea muy bueno en todo tipo de actividades y propuestas. Todos tenemos más habilidades en algún aspecto que en otro. Por lo que he introducido apartados abiertos en la carpeta para que el estudiante pueda demostrar sus habilidades adquiridas con más libertad.

El incremento de no presentados en este caso tiene una clara explicación. La mayoría del colectivo de estudiantes de esta asignatura está insertado de forma total o parcial en el mundo laboral, lo cual implica que no siempre pueden asistir a clase, o hacer las entregas correspondientes. Inicialmente hacen el propósito de conseguirlo pero durante el proceso algunos se ven obligados a abandonar por el camino.

3. CIENCIAS SOCIALES Con la introducción de CAES disminuyen considerablemente los Y SU DIDÁCTICA I suspensos hasta desaparecer al cabo de 2 años.

La diferencia de los resultados en los años de aplicación de CAEs no es nada significativa desde el punto de vista estadístico.

En los cursos de aplicación de carpetas se denota un traspaso de alumnos a notas superiores, teniendo el último curso estudiado un $70 \%$ de notables y un $25 \%$ de sobresalientes.

Comentario del profesor: Queda claro que los resultados han mejorado muchísimo ya que se ha logrado disminuir paulatinamente los estudiantes no presentados, y han desaparecido los suspendidos. En los primeros años de aplicación de carpeta los estudiantes mayoritariamente se situaban en el aprobado (59\%), unos pocos en el notable (14\%) y unos menos en el sobresaliente (7\%), por lo que se empezaron a introducir pequeños cambios en la carpeta para poder recuperar la excelencia, y los resultados han sido muy positivos, ya que en el último año de aplicación las notas fueron de un residuo del 3,5\% de aprobados, la mayoría (69\%) de notables y del $24 \%$ de sobresalientes. Una de las medidas que se han ido incorporando en la CAES para 'recuperar' la excelencia ha sido un proceso de autoevaluación que implicaba procesos metacognitivos, es decir, los estudiantes no solo debían ir 'entregando' evidencias de su aprendizaje, sino que debían valorar su proceso y progreso, mediante la elaboración de un texto argumentativo donde ilustraran su evolución en el aprendizaje, su implicación y su esfuerzo. Además, se incluyó un apartado optativo que de ser realizado completamente, en el tiempo estipulado y siguiendo las orientaciones previas, podía convertirse en el elemento diferenciador entre el sobresaliente y la matrícula de honor. Esta decisión fue tomada ante la situación de que los excelentes y las matrículas habían desaparecido, a pesar de existir estudiantes realmente brillantes. 
MAGDA CAYÓN COSTA Y MARTA FUENTES AGUSTÍ

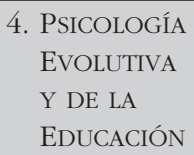

Entre el primero y el segundo año tras la implantación aún se dan diferencias en los resultados significativas por encima del $99 \%$, pero entre el segundo y el tercer año no.

Los resultados son muy similares, desaparecen los suspendidos, hay un porcentaje muy bajo de no presentados y la mayoría de alumnos se reparte entre el notable y el sobresaliente.

Comentario del profesor. Al introducir la carpeta se da la posibilidad al estudiante de ir mejorando su trabajo de forma continua tras la retroalimentación evaluativa, lo que facilita que el global de los estudiantes se sitúe en las mejores calificaciones. Esta retroalimentación ha sido mucho más intensa en los últimos años, incorporando la defensa pública durante el proceso, lo que provoca la participación en la evaluación de todos y cada uno de los estudiantes del grupo clase. Cabe decir también que los criterios y argumentos del estudiante que asume el rol en las coevaluaciones son elemento evaluable. Esto propicia que el estudiante se implique más con lo cual aumenta su aprendizaje, lo que provoca una disminución de los estudiantes no presentados y desaparecen los suspendidos. La estructura inicial de la carpeta ha variado levemente pero sí se ha profundizado en propiciar el diálogo y la reflexión grupal e individual. Respecto la disminución de los estudiantes no presentados se debe a que cuando uno inicia la carpeta le cuesta renunciar a ella, es consciente de sus conocimientos previos, sus metas, sus avances y posibles aplicaciones prácticas de los logros alcanzados. A demás es visible el proceso de evaluación y mejorable el producto. Se combina la rigurosidad con la flexibilidad y la implicación es un valor capital.

\section{ATENCIÓN \\ A LA PRIMERA \\ INFANCIA}

En este caso los suspendidos y aprobados desaparecen, situándose entre el $70-75 \%$ en el notable y un porcentaje residual en el sobresaliente.

Se consigue disminuir los NP del $26 \%$ al $17 \%$. Se recupera un poco la excelencia pero más del $70 \%$ de los estudiantes se sitúan en el notable sin alcanzar el sobresaliente.

No se da significación estadística en la diferencia de los resultados.

Comentario del profesor. La no significatividad es totalmente lógica ya que desde el principio se ha utilizado carpeta propiamente dicha. A pesar de esto, la carpeta aplicada en el primer año de estudio es muy distinta a la carpeta del último año, no en su esencia, pero sí en formatos, por el hecho de la introducción de novedades continuas, etc. La disminución de los no presentados es debida básicamente a la mayor implicación del estudiante en su propio proceso de aprendizaje y la corresponsabilidad que esto implica. Las novedades continuas tienen que ver con la aplicación de medidas de acompañamiento a los estudiantes a través de tablas de criterios de evaluación entre iguales o de autoevaluación, la aplicación de herramientas de registro de las actividades que forman parte de la carpeta y de la revisión de las metodologías docentes entre un curso y otro.

\section{Resumen de los principales resultados anteriores:}

1. Los resultados cuantitativos son dispares a nivel de que en unos casos los resultados académicos han sido significativamente mejores desde el punto de vista estadístico, pero en otros casos no. Pero los resultados cualitativos sí que son comunes: reducción de los estudiantes no presentados 
MAGDA CAYÓN COSTA Y MARTA FUENTES AGUSTÍ

INCIDENCIA DE LAS CARPETAS DE APRENDIZAJE EN EL RENDIMIENTO ACADÉMICO DE LOS ESTUDIANTES UNIVERSITARIOS

y aumento de las notas de mayor rango. Las causas que provocan esta evolución positiva también parecen bastante claras y comunes: el hecho de implicar al estudiante en los procesos de evaluación, coevaluación y autoevaluación ha provocado el desarrollo de procesos metacognitivos de valoración de su proceso y progreso en la asignatura, así como la introducción de las medidas de acompañamiento como las tablas de criterios de evaluación, las presentaciones públicas de las carpetas individuales, y la posibilidad de añadir aspectos optativos para enriquecer la carpeta personal.

2. El uso de la CAES proporciona alta satisfacción al profesorado implicado ya que, con independencia de las notas finales, se mantiene un diálogo asiduo con el alumno de manera que el grado de conocimiento que el docente tiene sobre el nivel de aprendizaje que el estudiante ha adquirido es muy elevado.

3. Al inicio de la aplicación de la CAES se tiende a perder la excelencia en los alumnos, por lo que el profesorado introduce herramientas en la misma carpeta para poder recuperar a esos alumnos sobresalientes.

4. En los últimos cursos académicos, y coincidiendo con la implementación total de los grados en el nuevo espacio europeo, se denota un incremento de asignaturas que hacen mayor uso de metodologías activas, de evaluación continuada, etc. Lo que lamenta el profesorado experto en el uso de las CAEs es que, en muchos casos, a metodologías basadas en entregas parciales, evaluaciones continuadas, etc., se las denomina carpetas y no lo son en su esencia.

5. El profesorado entrevistado comenta que el hecho de que se empiece a incrementar la implantación de las carpetas o pseudocarpetas está provocando una pérdida de calidad de las mismas.

\section{DisCUSIÓN Y CONCLUSIONES}

En respuesta al objetivo general planteado, se puede afirmar que, con la aplicación de la metodología docente Carpetas de Aprendizaje en Educación Superior (CAES) en los casos estudiados, los resultados académicos de los estudiantes mejoran claramente.

Atendiendo a los objetivos específicos:

1. En primer lugar y atendiendo a los cambios en los rendimientos académicos los resultados son que se ha producido un aumento estadísticamente significativo en los cursos de implantación de CAEs que en los anteriores utilizando otras metodologías. Se reducen drásticamente los suspendidos, y el nivel mayoritario se sitúa en el notable. Se experimenta una pérdida de excelencia que el profesor combate con la introducción de herramientas y posibilidades en la propia carpeta que permita al estudiante 
MAGDA CAYÓN COSTA Y MARTA FUENTES AGUSTÍ

INCIDENCIA DE LAS CARPETAS DE APRENDIZAJE EN EL RENDIMIENTO ACADÉMICO DE LOS ESTUDIANTES UNIVERSITARIOS

demostrar sus capacidades para la obtención de la máxima calificación. Y finalmente los alumnos no presentados, en algunos casos aumentan y en otros disminuyen. Los que aumentan, básicamente es porque nos encontramos con colectivos que trabajan y les resulta complicado poder asistir a clase, hacer las entregas puntualmente, etc. En cambio, en los colectivos de estudiantes que sí pueden asistir regularmente a clase y seguir la asignatura, los no presentados disminuyen claramente debido a la implicación que adquieren de forma inducida con su propio aprendizaje, y a la corresponsabilidad en la evaluación.

2. En segundo lugar, y atendiendo a los cambios en los rendimientos académicos durante los años en que se ha implantado la CAEs, los resultados no son tan claros. Hay años y asignaturas que sí mejoran pero en otros casos no existen diferencias estadísticamente significativas. A nivel cualitativo sí que hay unanimidad entre estudiantes y profesorado en que la mejoría en el aprendizaje se da utilizando la CAES. Sobre todo porque implica que el hecho de implicar al estudiante en los procesos de evaluación, coevaluación y autoevaluación ha provocado el desarrollo de procesos metacognitivos de valoración de su proceso y progreso en la asignatura, así como la introducción de las medidas de acompañamiento como las tablas de criterios de evaluación, las presentaciones públicas de las carpetas individuales y la posibilidad de añadir aspectos optativos para enriquecer la carpeta personal.

Con el uso de la CAES, el profesorado se asegura un mejor aprendizaje de sus alumnos ya que se lleva a cabo un mayor seguimiento del progreso del estudiante, se comparten las evaluaciones, se reflexiona periódicamente sobre el proceso, etc., pero sobre todo el profesor se asegura de que el estudiante haya adquirido las competencias específicas y transversales trabajadas.

En los casos en que la metodología docente anterior a la CAEs era similar, los resultados académicos no difieren demasiado, pero lo más importante es que con la carpeta, a diferencia de otras metodologías, el profesor puede saber qué, cómo y cuándo ha aprendido el estudiante.

Gracias al diálogo reflexivo que se establece continuamente con los estudiantes, el profesorado obtiene mucha información para su propia mejora continua, tanto a nivel personal como metodológico.

Los principales cambios observados al utilizar la CAES son los siguientes:

- Aumenta el grado de complicidad entre el profesor y los estudiantes y reduce el aislamiento.

- Aumenta el grado de implicación del estudiante situándose en el centro del proceso de enseñanza y aprendizaje.

- Obliga al estudiante a mantener "el trabajo al día" con todas las ventajas que ello conlleva. 
MAGDA CAYÓN COSTA Y MARTA FUENTES AGUSTÍ

INCIDENCIA DE LAS CARPETAS DE APRENDIZAJE EN EL RENDIMIENTO ACADÉMICO

DE LOS ESTUDIANTES UNIVERSITARIOS

- Ayuda al estudiante a responsabilizarse de su formación al mismo tiempo que le genera una disonancia cognitiva en torno al papel activo del aprendiz.

- Pide tiempo, dedicación y constancia, y muchas veces el estudiante no está dispuesto a pagar el "precio" que esto supone.

- Pide ciertas competencias y habilidades que no siempre el estudiante tiene adquiridas.

- Mejora la comunicación entre profesorado-alumnado.

- Facilita la labor docente, ya que agiliza la gestión de la información y la organización de la asignatura en general.

- Aumentan los elementos y argumentos para una evaluación justa del estudiante.

- Facilita la evaluación formativa, sumativa y continua del estudiante por lo que hace más transparente el proceso de enseñanza y el sistema de evaluación.

Por todo lo anterior, proponemos la aplicación de la CAEs en las asignaturas universitarias ya que incide positivamente en la calidad docente, tanto a nivel de mejorar los resultados académicos como a nivel de optimizar el proceso de aprendizaje.

Debido a la constatación de que a través de portfolio los estudiantes aprenden más y mejor y que cuantos más profesores apliquen este tipo de metodología unilateralmente puede suponer una carga de trabajo excesiva, proponemos que la tendencia en las titulaciones debería estar orientada a desarrollar portfolios por áreas o por titulaciones de forma que las competencias transversales se trabajen pero sin duplicarse. Somos conscientes de que esta propuesta implica un grado elevadísimo de coordinación de las materias y sobre todo homogeneización de criterios entre los docentes, que no siempre puede ser fácil de conseguir.

\section{REFERENCIAS BIBLIOGRÁFICAS}

- (2009). Monográfico VIII.- Portafolios electrónicos y educación superior en España. Revista de Educación a Distancia.

Alegret, M.; Laguna, J. C.; Vázquez, M. y Sánchez, R. M. (2006). La carpeta de aprendizaje: un sistema alternativo de evaluación en la asignatura de farmacología de las enfermedades metabólicas. En Actas del IV Congreso Internacional Docencia Universitaria e Innovación. Barcelona, 5-7 de julio.

Barberà, E. (2005). La evaluación de competencias complejas: la práctica del portafolio. Educere. Revista Venezolana de Educación, año 9, n.․ 31.

Barrett, H. C. (2004). Electronic portfolios as digital stories of deep learning. Recuperado de http://electronicportfolios.org/digistory/epstory.html.

Blanch, S. et al. (2010). Carpetes d'aprenentatge a l'educació superior: una oportunitat per repensar la docencia. Barcelona: Servei de publicacions de la Universitat Autònoma de Barcelona. 
MAGDA CAYÓN COSTA Y MARTA FUENTES AGUSTÍ

INCIDENCIA DE LAS CARPETAS DE APRENDIZAJE EN EL RENDIMIENTO ACADÉMICO DE LOS ESTUDIANTES UNIVERSITARIOS

Blanch, S. y Fuentes, M. et al. (2009). Relaciones entre aprendizaje, cognición y tecnologías en la construcción del e-portafolio. RED, Revista de Educación a Distancia, 8. Recuperado de http://www.um.es/ead/red/M8/.

Bia, A. (2005). El portafolio del discente como método de trabajo autónomo. En M. Á. Martínez Ruiz y V. Carrasco Embuena (Eds.). Investigar en diseño curricular. Redes de docencia en el Espacio Europeo de Educación Superior. Universidad de Alicante. Marfil.

Brookfield, S. D. (1995). Becoming a critically reflective teacher. San Francisco: Jossey-Bass.

Castro Quitora, L. (2002). El portafolio de enseñanza como herramienta y texto para la reflexión pedagógica. Revista Perspectiva Educativa, 3, 9-21.

Cerioni, M. (1997). Propuestas de evaluación metacognitiva. Recuperado de http://www. monografias.com/trabajos32/propuestas-evaluacion-metacognitiva/propuestas-evaluacion-metacognitiva.shtml.

Chatel, R. G. (2001). Portfolio development: some considerations. http://www.eric.ed.gov/ ERICDocs/data/ericdocs2sql/content_storage_01/0000019b/80/19/86/7f.pdf. Descargado el día 26 de noviembre de 2007.

Colén, M. T.; Giné, N. e Imbernon, F. (2006). La carpeta de aprendizaje del alumnado universitario: la autonomía del estudiante en el proceso de aprendizaje. Barcelona: Octaedro-ICE.

Farr, R. y Tone, B. (1994). Portfolio and performance assessment. Helping students evaluate their progress as readers and writers. Fort Worth: Harcourt Brace College Publishers.

Fuentes, M. y Oliver, S. (2008). De la definició a l'aplicació de la carpeta d'aprenentatge. En S. Blanch et al. Carpetes d'aprenentatge a l'educació superior: una oportunitat per repensar la docencia (pp. 11-24). Barcelona: Servei de publicacions de la UAB. [Publicación traducida en 2010 al español e inglés].

Fuentes, M.; Oliver, S. et al. (2009). Development and assessment of the student's skills by means of the use of virtual learning files (VLFs). En M. Martínez y E. Añanos (Coords.). Innovative teaching at the UAB in experimental and technological sciences and in health sciences (pp. 203-212). Barcelona: Servei de publicacions de la UAB.

Gimeno, X.; González, N. y Santiveri, N. (2008). L'índex de la carpeta d'aprenentatge. En S. Blanch et al. Carpetes d'aprenentatge a l'educació superior: una oportunitat per repensar la docencia (pp. 25-42). Barcelona: Servei de publicacions de la UAB. [Publicación traducida en 2010 al español e inglés].

Hacker, D. J. (1998). Definitions and empirical foundations. En D. J. Hacker, J. Dunlosky y A. C. Graesser (Eds.). Metacognition in educational theory and practice. Nueva Jersey: Lawrence Erlbaum Associates Publishers.

Hernández Fernández, A. (2005). Un portafolio universitario: hacia una formación individualizada, activa, reflexiva y autorregulada. Santander: Tratamiento Gráfico del Documento.

Johnson, D. W.; Johnson, R. T. y Smith, K. A. (1991). Active learning: Cooperation in the College Classroom. Edina, MN: Interaction Book Company.

Johnson, R. S.; Mims-Cox, J. S. y Doyle-Nichols, A. (2006). Developing portfolios in education. A guide to reflection inquiry, and assessment. Londres: Sage Publications.

Klenowski, V. (2004). Desarrollo de portafolios para el aprendizaje y la evaluación. Madrid: Narcea.

Marby, L. (1999). Portfolios plus: A critical guide to alternative assessment. Thousand Oaks, California: Corwin Press, Inc. 
MAGDA CAYÓN COSTA Y MARTA FUENTES AGUSTÍ

INCIDENCIA DE LAS CARPETAS DE APRENDIZAJE EN EL RENDIMIENTO ACADÉMICO DE LOS ESTUDIANTES UNIVERSITARIOS

Martínez, C.; Bordons, G.; Guarro, B. y Manuel, J. (2006). Aprenentatge reflexiu i autònom aplicat a la docència de la llengua catalana: l'aplicació del portafolis. En Actas del IV Congreso Internacional Docencia Universitaria e Innovación. Barcelona, 5-7 de julio.

Pozo, J. I. y Monereo, C. (Eds.) (1999). El aprendizaje estratégico. Madrid: Santillana.

Sunstein, B. S. y Lovell, J. H. (Eds.). (2000). The portfolio standard. How students can show us what they know and are able to do. Portsmouth: Heineman.

Tobin, B. (1994). Literacy portfolios: reflecting on life's journey or learning: explorations and personal interpretations. EUA.

Ugalde, A. y López, R. (1995). El portafolio: en zapatos de estudiante. Recuperado de http:// www.una.ac.cr/bibliotecologia/boletinbiblioteca/1995/Elportafolio.doc.

Villar, L. M. (2004). Manual para la excelencia en la enseñanza superior. McGraw-Hill Interamericana de España.

Villar, L. M. y Alegre, O. M. (2005). Evaluación del aprendizaje de capacidades curriculares y didácticas: carpetas de actuaciones del profesorado. Enseñanza, 23, 423-449.

- (2008). Measuring Faculty Learning in Curriculum and Teaching competence (CTC) Online Courses. Interactive Learning Environments, 16 (2), 169-181. http://dx.doi. org/10.1080/10494820701365937

Watkins, C. (2001). Learning about learning enhances performance. NSIN Research Matters 13.

Zubizarreta, J. (2004). The learning portfolio. Reflective practice for improving student learning. Bolton, Massachusetts: Anker. 